\title{
Globalización, mercados y el futuro de las pesquerías*
}

\section{Globalization, Markets and the Future of Fisheries}

\section{Globalisation, marchés et l'avenir de la pêche}

\section{Carmen Pedroza-Gutiérrez**}

Recibido: 2014-03-20 // Aprobado: 2014-05-10 // Disponible en linea: 2014-09-10

Cómo citar este artículo: Pedroza-Gutiérrez, C. (2014). Globalización, mercados y el futuro de las pesquerías. Ambiente y Desarrollo, 18(35), 71-82. http://dx.doi.org/10.11144/Javeriana.AyD18-35.gmfp

doi:10.11144/Javeriana.AyD18-35.gmfp

\section{Resumen}

Este trabajo presenta una breve reflexión acerca de la situación de deterioro de la pesca de captura y las tendencias de consumo de los productos pesqueros. Considera algunos de los principales aspectos que caracterizan los mercados de estos productos a nivel global, entre ellos la expansión de los mercados pesqueros y el aumento del consumo, resultado de las innovaciones tecnológicas y la mejora en los canales de distribución, lo cual está teniendo impactos negativos en la pesca de captura y causa una crisis de abastecimiento de este tipo de productos pesqueros. Crisis que de acuerdo con algunos científicos pesqueros, de continuar las mismas tendencias, existe el riesgo de un colapso global de pesquerías.

Palabras clave: pesca; mercados; consumo; globalización

Este artículo es de reflexión y parte de resultados de investigación desde una perspectiva analítica, basándose en fuentes originales.

** Doctora en Socioeconomía del Desarrollo, investigadora de tiempo completo, Unidad Académica de Estudios Regionales (UAER) de la Universidad Nacional Autónoma de México. Correo electrónico: pedrozacarmen@yahoo.com 


\begin{abstract}
This work presents a short reflection on the decaying situation of capture fisheries and the consumption tendencies of fish products. The work considers some of the main aspects that characterize the markets of these products on a global level, including, the expansion of fishing markets and the increase of consumption, as a result of technological innovations and the improvement of distribution channels. This is having a negative impact on capture fisheries activities and causes a supply crisis of this type of fishing products. This crisis, according to some fishing scientists, provided the same tendencies continue, poses the risk of a global collapse of fisheries.
\end{abstract}

Keywords: fishing; markets; consumption; globalization

\title{
Résumé
}

Ce travail présente une brève réflexion de la situation de détérioration de la pêche de capture et les tendances de consommation des produits de la pêche. On considère parmi les principaux aspects quelques 'un qui caractérisent les marchés de ces produits au niveau globale, parmi eux l'élargissement des marchés de la pêche et l'augment de la consommation, à cause des innovations technologiques et l'amélioration dans les chaînes de distribution, cela a des impacts négatifs dans la pêche de capture et cela cause une crise d'approvisionnement de ce type de produits de la pêche. La crise que selon les scientifiques pêcheurs de continuer avec les mêmes tendances, risque de provoquer un collapse globale de la pêche.

Mots clés : pêche; marchés; consommation; globalisation 


\section{Introducción}

La pesca es una de las primeras actividades productivas llevadas a cabo por los seres humanos (Lackey, 2005). Es por esto que hasta la fecha el pescado ha estado presente dentro del comercio global de alimentos y su consumo ha contribuido como una fuente de proteína importante, principalmente para sociedades costeras o comunidades rurales que habitan cerca de cuerpos de agua continentales. Sin embargo, las dinámicas de su producción, distribución y consumo han tenido giros importantes en las últimas decadas.

En la actualidad, en un mundo afectado y transformado por la tecnología y la comunicación, que han logrado unir mercados, sociedades y culturas, la actividad pesquera es partícipe de estos fenomenos. Esto se ve reflejado en pesquerías artesanales, llevadas a cabo de forma tradicional, poco tecnificadas, asociadas a baja productividad y autoconsumo, y con canales de distribución locales (FAO, 2005). Posteriormente, conforme van apareciendo innovaciones y mejoras tecnológicas, en términos de conservación, artes de pesca, transporte y procesamiento, se convierten en pesquerías comerciales con una amplia distribución (Anderson, 2003; Kurien, 2005). Lo cual incentiva la expansión de los mercados y el aumento del consumo de productos pesqueros, principalmente en los países desarrollados. Consumo facilitado por una mayor disponibilidad (en términos de cantidad y variedad) de productos pesqueros y por el incremento del poder adqusitivo, además del aumento de la población (FAO, 2014; Fernández-Polanco, 2012). Es decir, se come más pescado porque hay más personas, pero también porque hay mayor cantidad y variedades disponibles.

Hoy en día la comercialización de productos pesqueros está compuesta por más de cuatro mil especies de organismos acuáticos y plantas (Lackey, 2005). Además de que existen más de ochocientas especies de peces, crustáceos y moluscos con importancia comercial (Anderson, 2003), hecho acompañado del incremento exponencial en el volumen de producción de productos pesqueros provenientes de la acuacultura (Fernández Polanco, 2012). Todo esto conforma el conjunto de mercancias que, dentro del marco de la globalización de las pesquerías, se ofertan en los mercados de productos pesqueros.

La expansión de estos mercados cuenta con algunos aspectos positivos, ya que muchos consumidores ahora pueden adquirir especies a las que anteriormente nunca hubieran podido tener acceso (Clover, 2006). Sin embargo, para poder satisfacer el incremento en el consumo se requieren mayores volúmenes de captura, lo cual ha resultado en sobrepesca, y esta, a su vez, lleva a la escasez a una de cada cuatro pesquerías comerciales del mundo (Mullon et ál., 2005), o bien a que una tercera parte del stock pesquero se reporte como sobrepescado (FAO, 2008; Hilborn, 2012).

Considerando lo anterior, este artículo tiene como objetivo hacer una reflexión sobre el marco general que caracteriza la situación actual de producción y consumo de la actividad pesquera. Para lograr este objetivo se presentan, en primera instancia, las características generales del consumo de productos pesqueros a nivel mundial, seguido esto de las consecuencias de este consumo, terminando con una reflexión final sobre algunas de las soluciones propuestas para aminorar el impacto generado por el aumento de la producción y el consumo. La metodología utilizada tiene como base la recopilación de información bibliográfica, además de la utilización y consulta de las bases de datos estadísticos de la Organización de las Naciones Unidas para la Alimentación y la Agricultura (FAO en su sigla en inglés), que recopilan el Estado y las tendencias de la producción y el consumo de productos pesqueros a nivel mundial.

\section{Resultados}

\section{Los mercados de productos pesqueros en el contexto globalizatorio}

El consumo de pescado a nivel mundial ha tenido sin duda un giro relevante. Mientras que en países como Camboya, Bangladesh, Indonesia o Senegal ha sido tradicionalmente la principal fuente de proteínas, para los consumidores occidentales se está convirtiendo en un bien cuyo consumo se ha agregado a los nuevos regimenes alimenticios. Esto se debe, por una parte, a que el pescado por 
ser un alimento sano forma parte de las dietas recomendadas por nutriólogos, como una solución a los crecientes problemas de obesidad que enfrenta la sociedad globalizada, o bien por su contenido en proteínas bajas en grasa, vitaminas y ácidos grasos omega 3, que reducen el peligro de ataques cardiacos y derrames cerebrales y retardan la artritis y la osteoporosis (Kris-Etherton et ál., 2002). Por otra parte, también es cierto que existen países como España, Portugal, Islandia, Noruega o Japón que tradicionalmente son considerados como países de tradición pesquera. Esto implica tener consumidores especializados en estos productos porque cuentan con una mayor tradición y experiencia en la pesca de captura y, por lo tanto, una parte importante de su cultura gastronómica se ha desarrollado en lo relativo al consumo de productos pesqueros (Fernández-Polanco, 2012). Estos han sido algunos de los aspectos que han incentivado el aumento de su consumo (Clover, 2006).

Por su parte, la faO (2012a), en su publicación El estado mundial de la pesca y la acuacultura en el mundo 2012 confirma que en los últimos cincuenta años el suministro mundial de productos pesqueros ${ }^{1}$ destinados a consumo humano ha tenido un incremento mayor al de la población mundial (1,7\% anual), con una tasa de crecimiento del 3,2\% anual entre 1961 y el 2009. En la figura 1 se puede observar esta evolución, ya que en la decada de los sesenta el consumo per cápita era de 9,9kg y en el 2009 llegó a 18,4 $\mathrm{kg}$. Lo cual indica que se consume más pescado, no solo por el incremento poblacional y porque un mayor número de personas lo ha agregado a sus dietas, sino también porque gracias al aumento en la variedad y volumen de productos pesqueros disponibles en el mercado de alimentos los consumidores tradicionales de pescado también pueden comer una mayor cantidad y variedad de estos productos.

Figura 1. Consumo mundial de productos pesqueros

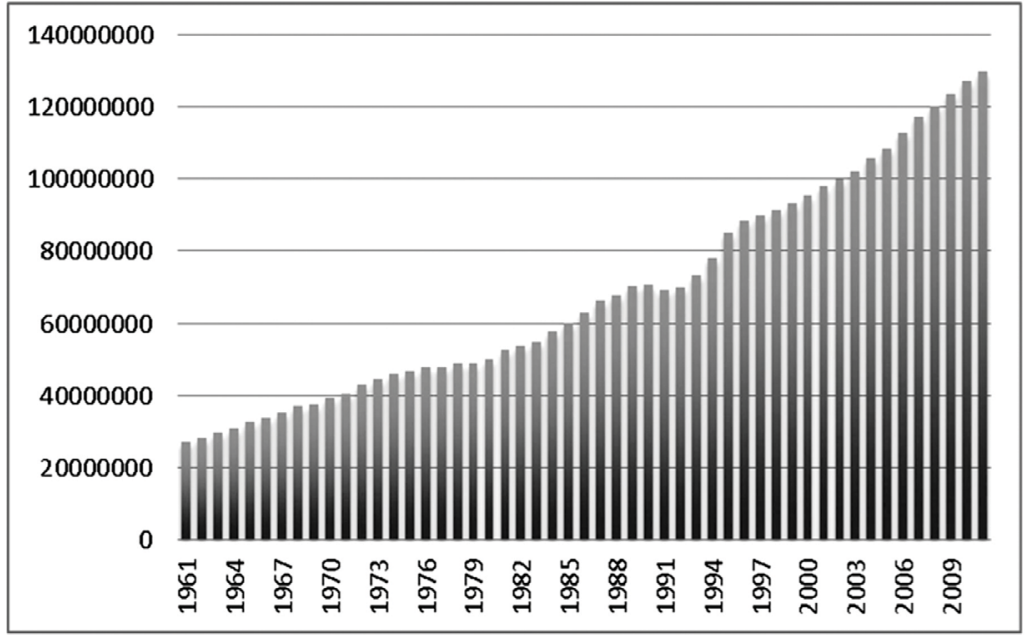

Fuente: FishStat, elaboración propia

Así, el incremento en el número de consumidores y la tradición son variables importantes que han incentivado el consumo de pescado. El consumo más alto de pescado en el 2009 se registró en Asia, con 85,4 millones de toneladas (20,7 kg per cápita), mientras que el menor consumo se dio en África con 9,1 millones de toneladas y 9,1 kg per cápita (FAO, 2012a). En Asia se encuentran algunos de los mayores consumidores de pescado a nivel mundial: China, Japón y las Islas Maldivas. Estos países tuvieron un consumo per cápita en el 2012 de 33,5, 51,7 y $164 \mathrm{~kg}$, respectivamente (FAO, 2012a). Sin embargo, en estos casos además de la tradición, el tamaño de la población hace la diferencia, ya que mientras China y Japón tienen 1368’ 440000 y 127’319000 de habitantes, respectivamente, las Islas Maldivas solo tienen 332000 habitantes. En el caso

1 Incluyendo pesca de captura y acuicultura. 
de China, el aumento en el consumo también se ha dado por el incremento de los ingresos (Clarke, 2009), y en el caso de Japón al ser una isla el pescado siempre ha formado parte de su tradición gastrónomica (Bestor, 2004). Sin embargo, el incremento en el consumo de carne está provocando una baja en el consumo de pescado (FAO, 2012b; Worldfishing and Aquaculture, 2013). Además de China y Japón, el país con mayor consumo de productos pesqueros es Estados Unidos (22 kg) y como región la Unión Europea (22 kg) (FAO, 2012b, 2014; Fernándes-Polanco, 2012), contando también con una cantidad importante de población (Estados Unidos, 314’ 912000 y la Unión Europea, 741’274 000).

Los factores que han hecho posible el aumento en el consumo, y a su vez han dado lugar a la expansión de los mercados a nivel regional y mundial, han tenido que ver con una distribución más eficiente y la innovación e implementación de diferentes formas de tecnológia (Anderson, 2003; FAO, 2007). En términos de conservación, el hielo, el invento del enlatado y los sistemas de refrigeración hicieron posible la mejor conservación del pescado para expandir su distribución; el GPs ha permitido la localización de grandes cardúmenes en diferentes zonas marinas, y la expansión carretara y la invención de diferentes formas de transporte facilitan las exportaciones intercontinentales (Anderson y Martínez-Garmendia, 2003).

Por otra parte, las nuevas tecnologías también han sido responsables del incremento en la producción acuícola. Dada la falta de capacidad de la pesca de captura para poder satisfacer la demanda, la acuacultura ocupa cada vez un lugar más importante en la producción pesquera. Así, en el 2012 esta actividad aportó un $49 \%$ del total de la producción pesquera mundial (FAO, 2014). Esto ha dado pie a una disminución de los precios, principalmente en especies como la tilapia y el bagre pangasius que forman parte de las especies con mayor volumen de producción en la actividad acuícola (Fernándes-Polanco, 2012). Esto a su vez ha provocado una reorganización de los mercados y formas de consumo de pescado. El notable incremento de los productos acuícolas ha dado lugar a la creación de nuevos mercados, ya que es posible que los productos pesqueros lleguen a un mayor número de consumidores. Hoy en día diferentes productos pesqueros (pesca de captura marina, continental y acuicultura) abastecen diferentes segmentos del mercado que pueden ser desde bienes de lujo con compradores especializados y de alto poder adquisitivo, como Japón, Noruega, Estados Unidos o Islandia, hasta mercados rurales con consumidores de bajo poder adquisitivo altamente dependientes de la pesca continental (Béné et ál., 2007).

Esta reorganización del comercio y el consumo de productos pesqueros se ve reflejada a través de las exportaciones en los mercados internacionales. Intercambios que se muestran en las estadísticas de la FAO (2014), en donde se afirma que el pescado se encuentran entre los productos alimenticios básicos más comercializados a nivel mundial. En el 2012 representó el 10\% de las exportaciones agrícolas totales. Se espera que estas exportaciones continúen con una tendencia hacia el alza, siendo los países en desarrollo los responsables de la mayor parte de ellas. En el 2010 dichos países abarcaron más del $50 \%$ de las exportaciones pesqueras en términos de valor y más del $60 \%$ en volumen (peso vivo) ${ }^{2}$ en los mercados mundiales. De ese $50 \%$, el $67 \%$ tuvo como destino países desarrollados.

Desde mediados de los noventa empezaron a incrementarse las importaciones de productos pesqueros tanto en Estados Unidos (figura 2) como en Japón (figura 3). Hoy estos son los mayores importadores de dichos productos, dependiendo de ellos en un 60 y $54 \%$, respectivamente, para su consumo de pescado (FAO, 2012a). Dado el incremento en la dependencia de las importaciones, y desde el colapso de las pesquerías de Nueva Inglaterra en los ochenta, los estadounidenses consumen más pescado importado que producido en el país (Clover, 2006). Japón con el mercado de productos pesqueros más grande del mundo, Tsukiji, y uno de los consumos per cápita más altos también, es altamente dependiente de las importaciones de productos pesqueros (Bestor, 2004). La Unión Europea, aunque con un consumo muy heterogéneo dependiendo del país de que se hable, es también uno de los importadores de productos pesqueros más importantes a nivel mundial, dado el aumento en el consumo y la disminución en la capacidad para satisfacer su propia demanda interna, debido al deterioro de sus pesquerías (Fernándes Polanco, 2012; FaO, 2014).

2 Peso total del producto en el momento de obtenerse de su medio natural. 
Figura 2. Exportaciones e importaciones de productos pesqueros en Estados Unidos

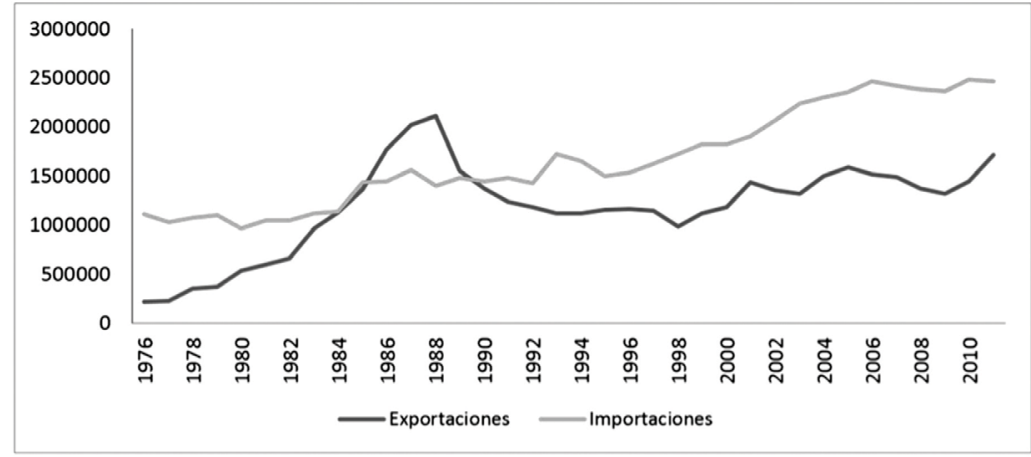

Fuente: FishStat, elaboración propia

Figura 3. Importaciones y exportaciones de productos pesqueros en Japón

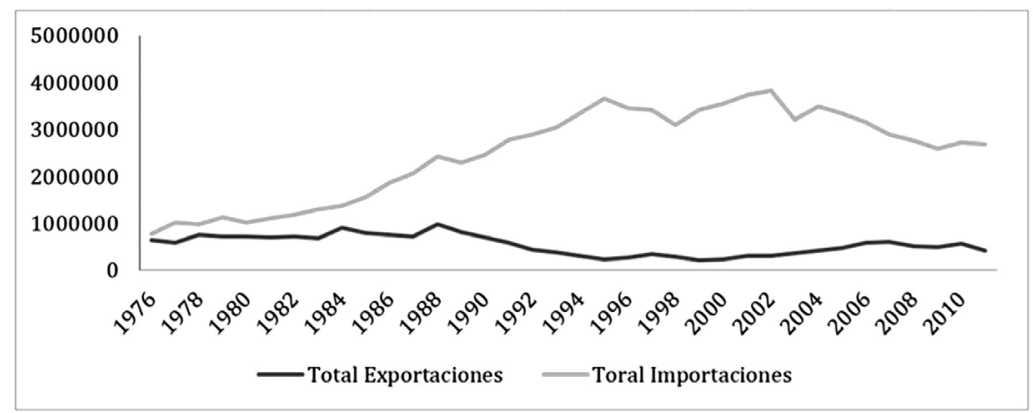

Fuente: FishStat, elaboración propia

El crecimiento en la dependencia de las importaciones para poder satisfacer el aumento del consumo interno se puede observar en las figuras 4 y 5 , en donde se muestra cómo las importaciones van en aumento, mientras que las exportaciones disminuyen. En el caso de la Unión Europea se observa un aumento exponencial de las importaciones a partir del 2005 (figura 4), hecho que coincide con el periodo en que China empieza a exportar masivamente productos pesqueros (figura 5).

Figura 4. Importaciones y exportaciones de productos pesqueros en la Unión Europea

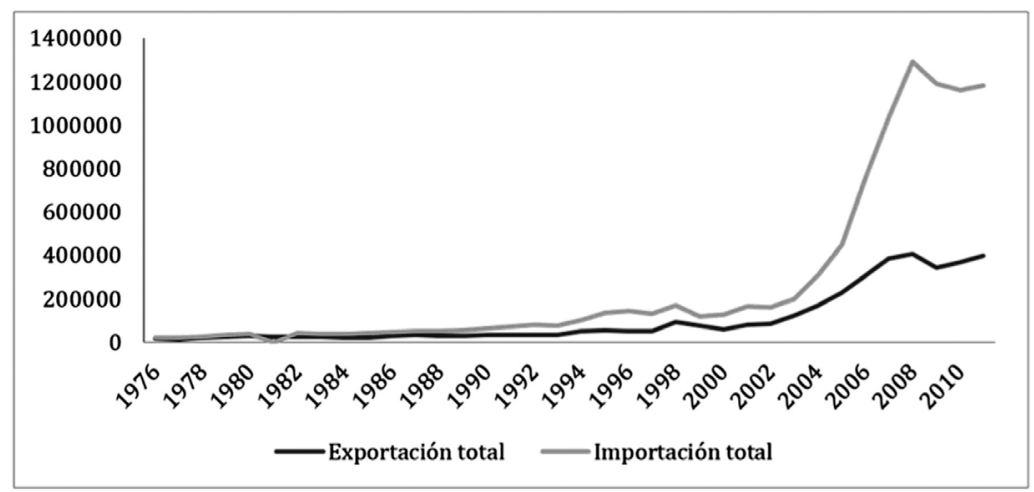

Fuente: FishStat, elaboración propia 
Otro de los países que han contribuido sustancialmente a la reorganización de los mercados de productos pesqueros es China, ya que desde el 2002 es el mayor productor y exportador a nivel mundial (Clarke, 2009). En el 2010, gracias a que sus exportaciones han crecido exponencialmente a partir de 1999, este país pudo contribuir con casi el 12\% de las exportaciones a nivel mundial (figura 5). Hoy también han aumentado notablemente sus importaciones (figura 5). Una de las razones que explican esta situación, además de la creciente demanda interna, es la reexportación de los productos pesqueros, ya que China importa materia prima y exporta productos transformados (Clarke, 2009). De esta manera, en el 2011 este país se convirtió en el tercer mayor importador de productos pesqueros (FAO, 2012a).

Figura 5. Exportaciones, importaciones y producción anual de productos pesqueros en China

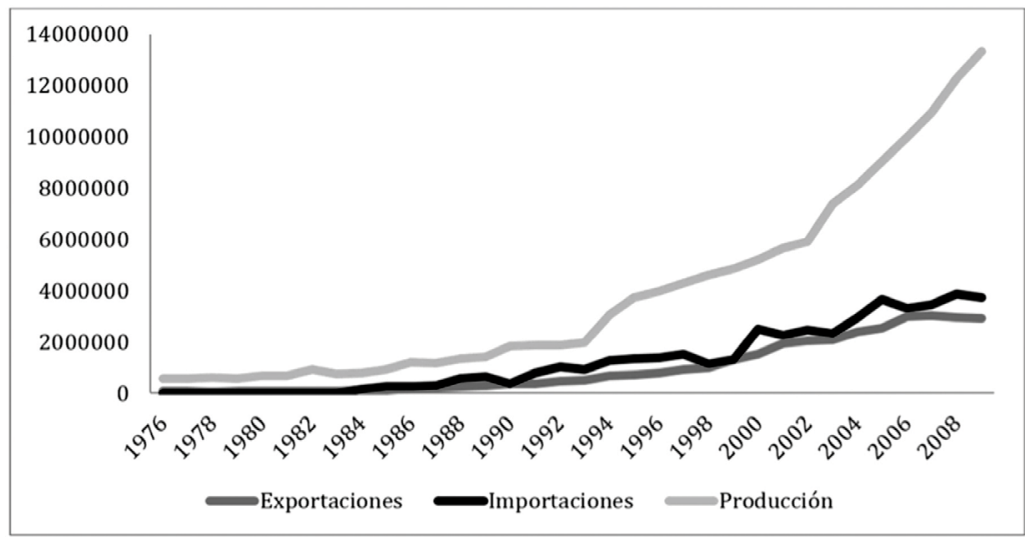

Fuente: FishStat, elaboración propia

Se observa así cómo mientras disminuye la producción de pesca de captura en Europa, Japón y Estados Unidos aumentan las importaciones. Esto indica que el incremento en el consumo de productos pesqueros en países desarrollados va acompañado de una disminución nacional de la producción de pesca de captura, lo cual está siendo reemplazado por importaciones. La FAO (2012b) supone que la dependencia de estos países de las importaciones de productos pesqueros provenientes de países en desarrollo ira en aumento.

\section{Discusión: consecuencias del incremento en el consumo de productos pesqueros y el futuro de las pesquerías}

Las diferentes consecuencias del incremento en el consumo y comercio de productos pesqueros pueden ser vistas desde diferentes perspectivas. Por una parte, existen argumentos que sostienen que el comercio de productos pesqueros causa pobreza y es una amenaza para la autosuficiencia alimentaria de países altamente dependientes de estos recursos. Por otra parte, se han elaborado explicaciones de cómo esta actividad puede ser generadora de riqueza, ingresos y desarrollo económico (Béné et al., 2010).

La perspectiva de que el comercio de productos pesqueros es fuente generadora de riqueza tiene como base los grandes flujos de exportaciones entre países en desarrollo y países desarrollados. Esto se refleja en que el valor de las exportaciones de productos pesqueros ha tenido aumentos significativos, ya que entre 1976 y el 2000 pasaron de 8000 millones de USD a 102000 millones de USD, y en el 2010 los países en desarrollo abarcaron más del 50\% del valor de las exportaciones (FAO, 2012a). Por esta razón, se argumenta que las exportaciones de estos productos se convierten en una fuente generadora de divisas para los países en desarrollo y que pueden ser utilizadas para pagos de deuda externa, o bien ser 
invertidas para contribuir a la economía local (Bostock et ál., 2004; Thorpe, 2004).

Sin embargo, existen argumentos que explican que la comercialización de productos pesqueros es causa de pobreza. Esto tiene como base la mala distribución de los ingresos provenientes de las exportaciones, que las rentas obtenidas por la extracción de los recursos no se consideran justas y el impacto que esto tiene sobre la autosuficiencia alimentaria de países altamente dependientes de sus recursos pesqueros (Béné et ál., 2010). Las exportaciones pueden tener un impacto negativo para la autosuficiencia alimentaria, al disminuir la oferta local de pescado en estos países (Wilson \& Boncoeur, 2008). Otro aspecto importante, además de la dependencia en aspectos nutricionales, es que esta actividad también es fuente generadora de empleos e ingresos (Béné et ál., 2007). Además de que hay una gran cantidad de personas que dependen de manera directa o indirecta de los recursos pesqueros, se calcula que en países en desarrollo el sector pesquero emplea entre 22 y 24 millones de pescadores, además de unos 60 o 70 millones de personas que trabajan en actividades derivadas de la pesca (transformación, distribución, comercialización) (Hall et ál., 2010).

Esto es, para poder exportar y satisfacer el incremento de la demanda de productos pesqueros, la lógica ha sido tratar de aumentar el volumen de pesca de captura. Para poder lograr esto, la tendencia ha sido ampliar la inversión para aumentar el esfuerzo pesquero ${ }^{3}$ y por lo tanto la oferta de pescado. Sin embargo, este fenómeno ha resultado en la sobrecapitalización del esfuerzo pesquero (Clover, 2006), sobrecapitalización con la que se intenta satisfacer la creciente demanda de productos pesqueros y que se lleva a cabo incrementado o mejorando la capacidad tecnológica para aumentar así los volúmenes de captura. Clover (2006) explica que esto tiene una correlación inversamente proporcional al volumen de captura, es decir, actualmente aunque se aumente el esfuerzo pesquero, esto no necesariamente implica mayores capturas; incluso en algunos casos los volúmenes de pesca son cada vez menores. Estudios como el de Pauly et ál. (2003) señalan que el volumen de desembarque está descendiendo aproximadamente 500000 toneladas métricas anualmente, desde que el volumen de captura alcanzó su máximo nivel a finales de los ochenta.

Esto se refleja en que a pesar del aumento en el esfuerzo pesquero, la producción mundial de pesca de captura marina ha permanecido estable desde finales de los años noventa (FAO, 2012a). Por esta razón, la acuacultura aporta cada vez mayores volúmenes para satisfacer la demanda mundial de pescado. Así, entre 1970 y 2000 la producción acuícola pasó de 3,9\% de la producción mundial a $27,3 \%$ y en el 2012 los peces comestibles provenientes de la acuacultura pasaron a ocupar el $42,2 \%$ del total de pescado producido (FAO, 2014).

El aumento en el consumo y las medidas para satisfacerlo han resultado en impactos negativos en los recursos pesqueros. Unos de los más comunmente mencionados son la sobreexplotación y la sobrepesca, lo cual esta resultando en el agotamiento de varios de los más importantes caladeros de peces a nivel mundial (Mullon et ál., 2005; Fernández Polanco, 2012; Hilborn, 2012). Mullon et ál. (2005) explican que entre 1950 y 2000 una de cada cuatro pesquerías han colapsado. Por su parte, la FAO (2008) reporta que un $80 \%$ de pesquerías comerciales están sobreexplotadas, agotadas o completamente explotadas.

Además de los ya mencionados problemas de sobrepesca, algunos de los artes de pesca, principalmente pesca de arrastre, dañan o destruyen el fondo marino, lo cual afecta directamente el hábitat que permite la reproducción de animales acuáticos (Díaz \& Rosenberg, 2008). Esto aunado al cambio climático y otras actividades antropogénicas, incentiva el deterioro de la pesca (Halpern et ál., 2008). Estos factores están comprometiendo la capacidad del océano como proveedor de alimentos, para mantener la calidad del agua y recuperarse de las perturbaciones (Worm et ál., 2006), aunque tal vez la mayor implicación de la destrucción de ecosistemas acuáticos son los servicios que proveen para la sociedad.

Considerando que existen varios elementos que incentivan el constante deterioro de las pesquerías,

3 Esfuerzo pesquero o de pesca: representa el número de artes de pesca de un tipo específico utilizado en los caladeros en una unidad de tiempo determinada (http:/www.fao.org/docrep/003/w4230s/w4230s09.htm). 
hay científicos que se han dado a la tarea de analizar y predecir el posible colapso a nivel global de las pesquerías para el 2048 (Worm et ál., 2006), o bien la destrucción total del hábitat y fondo marino para el 2050 (Pauly et ál., 2003), lo cual resultaría en un muy difícil mantenimiento de las pesquerías.

Así, mientras los científicos predicen un futuro poco alentador, los consumidores de Estados Unidos, la Unión Europea y Japón parecen estar dispuestos a pagar más por los peces de captura que quedan en el mar. El pescado se hace más caro en términos reales, conforme la demanda va en aumento. A medida que pasa el tiempo y aumenta la demanda de pescado, también aumenta el déficit de producción pesquera en estos países, el cual es abastecido con productos de países del Tercer Mundo. La Unión Europea y Estados Unidos, principalmente, gastan cientos de millones de pesos para comprar acceso a pesquerías en diferentes partes del mundo. La Unión Europea ha firmado varios acuerdos con países del oeste africano, y Estados Unidos con países del sureste asiático. En estos acuerdos se han cuestionado varios aspectos, uno de ellos es la sobreexplotación de recursos que ya se evidencia en algunos países como Angola y Senegal (Clover, 2006). Esto también favorece el argumento de que la comercialización de recursos pesqueros provoca pobreza y pone en juego la autosuficiencia alimentaria de algunos países en desarrollo, ya que los consumidores de los países más ricos adquieren estos productos reduciendo la oferta de alimentos para países en desarrollo.

Para los consumidores de países desarrollados, el dejar de comer pescado puede representar simplemente el cambiar un estilo de vida, mientras que para las personas de países en desarrollo puede llegar a tener implicaciones incluso de autosuficiencia alimentaria, reducción de ingresos y desempleo (Hall et ál., 2010; Béné et ál., 2010).

\section{Conclusión: posibles soluciones para aminorar el impacto de la produc- ción y el consumo}

Los científicos pesqueros, además de analizar el estado actual de las pesquerías y diseñar modelos que pueden predecir su colapso global bajo las condiciones actuales, también se dan a la tarea de sugerir cambios en las medidas de manejo de las pesquerías y en los mercados de consumo, para tratar de evitar o mitigar el colapso total de la actividad.

Una de las sugerencias que atañe a los consumidores es la de incentivar la responsabilidad y conciencia ambiental, y que el consumidor exija productos provenientes de pesquerías con sistemas de manejo más sustentables (Clover, 2006). La idea es controlar la demanda, para que a su vez las condiciones bajo las cuales esta se satisface se consigan de manera responsable y sustentable. Para esto han surgido propuestas de organizaciones no gubernamentales, como el Marine Stewardship Council (MSC), para la certificación de pesquerías y asegurar su sustentabilidad.

Otras sugerencias se enfocan en la implementación de políticas en los diferentes aspectos del manejo de pesquerías.

Una de las prácticas de manejo ampliamente sugeridas ha sido la disminución del esfuerzo pesquero y la implementación de un sistema de cuotas (Pauly et ál., 2003; Hall et ál., 2010; Barking \& De Sombre, 2013). Barking \& De Sombre (2013) han sido más específicos en sugerir que este se debe reducir de un 20 a $30 \%$ y redistribuirlo en los diferentes niveles tróficos. Así se podrá aminorar el fenómeno de substitución señalado por Clover (2006), en donde las especies grandes van siendo reemplazadas por peces más pequeños. Como parte de esto se especifica que hay que disminuir especialmente la pesca de arrastre y la captura incidental, ya que son las actividades que dañan el fondo marino e impactan directamente las poblaciones de peces.

Es necesario crear zonas marinas protegidas en donde se prohíba la pesca comercial (Pauly et ál., 2003; Hall et ál., 2010), pero donde además existan sistemas de monitoreo y programas para la reconstrucción de los principales stocks de peces. Barking y De Sombre (2013) llaman a esto un sistema de manejo global de pesquerías.

Dado el constante deterioro de las pesquerías y sus ecosistemas, y la falta de eficacia de los sistemas 
de manejo que se han aplicado, otra propuesta para tratar de asegurar la sostenibilidad de la pesca y la integridad de sus ecosistemas ha sido el enfoque ecosistémico. Este reconoce la necesidad de un mayor conocimiento sobre los ecosistemas, integrando los componentes sociales y económicos de la pesca y no solo la perspectiva biológico-pesquera (García et ál., 2003). El enfoque ecosistémico expresa la necesidad de que la ordenación pesquera debe orientarse a ecosistemas completos, para comprender tanto las dinámicas de las poblaciones pesqueras como su relación con los cambiantes factores ambientales (FAO, 1984). Debido a ello, debe enfocarse en los componentes de los ecosistemas dentro de un área geográfica, en una forma más holística que los sistemas de manejo anteriores (García et ál., 2003). Así, este enfoque se basa en principios entre los cuales destacan los siguientes: a) las pesquerías deberán manejarse limitando su impacto en el ecosistema, b) se deben mantener las relaciones ecológicas entre las especies asociadas y dependientes, c) las medidas de manejo deberán ser compatibles a través de la distribución del recurso, d) se deberá aplicar el principio precautorio, y e) la gobernanza deberá asegurar el bienestar de las personas y los ecosistemas (FAO, 2003, p. 15).

Finalmente, se propone reducir los subsidios (Pauly et ál., 2003) y eliminar la pesca ilegal, no reglamentada y no registrada (IUU, Iegal Ureported Uregulated fishing) (Barking \& De Sombre, 2013), actividades que forman el elemento clave de las problemáticas de la actividad pesquera, ya que gran parte de la sobrepesca se lleva a cabo dentro de un contexto de ilegalidad.

Sin embargo, Hall et ál. (2010) explican que para mantener el consumo per cápita actual se requieren 1,6 millones de toneladas más cada año, aumentando a 4,2 para el 2030. Para poder sostener este nivel de consumo es necesario mantener el volumen de captura e incrementar el nivel de producción de productos provenientes de la acuacultura. Sin embargo, el aumento de la producción acuícola también implica grandes cuestionamientos. Por una parte, está el impacto ambiental, y por otra, los socioeconómicos. Los principales impactos ambientales se han identificado como la pérdida o modificación del hábitat (p. ej., tala de manglar), captura excesiva de reproductores, introducción de especies exóticas, escape de las especies cultivadas, propagación de enfermedades, mal uso de químicos y antibióticos, liberación de desechos y dependencia de la pesca de captura (Primavera, 2006; Mialhe et ál., 2013). Los impactos socioeconómicos se han identificado en los costos sociales que implica la pérdida de hábitats como el manglar, o bien existen algunos casos de conversión de tierras, migración y desempleo rural (Bailey, 1988; Kautsky et ál., 1997; Mialhe et ál., 2013).

Hasta la fecha, las propuestas de manejo que se han probado en la escala nacional o regional, no han comprobado su eficiencia, ya que las pesquerías siguen estando sobreexplotadas. Tal vez una medida de manejo que pudiera aminorar la sobrepesca, y por lo tanto el daño a los ecosistemas marinos, sería aplicar regulaciones más estrictas y hacer mayor presión sobre su cumplimiento a las grandes transnacionales que tienen impactos importantes sobre la pesca. Como ejemplo se puede mencionar el caso de la empresa Mitsubishi y el atún azul. Esta empresa ha llegado a controlar entre el 35 y el $40 \%$ del stock de esta especie. Además, en el 2009 la captura permitida fue de 22000 toneladas anuales; sin embargo, se llegaron a pescar hasta 60000 . Gran parte de esta sobrepesca se ha atribuido a esta compañía, ya que al parecer su estrategia de acaparamiento va encaminada a beneficiarse de la escasez de esta especie, para después poder especular con sus precios (Clover, 2006; WorldFishing Today, 2009). En la medida en que se puedan controlar estos comportamientos de acaparamiento, y ejercer mayor presión sobre quienes pescan con métodos más dañinos, probablemente se tengan impactos positivos en la recuperación de algunas de las pesquerías en el mundo, como es el caso de los grandes pelágicos.

Agradecimientos: Investigación llevada a cabo gracias al apoyo del proyecto PAPIIT RR380212 de la UNAM. Así mismo, se reconoce la importancia de los comentarios y observaciones hechas por los revisores y el comité editorial para enriquecer el presente manuscrito. 


\section{Referencias}

Anderson, J. L. (2003). The International Seafood Trade. Woodhead Publishing: CRC Press.

Anderson, J. L. \& Martínez-Garmendia, J. (2003). Trends in International Seafood Trade, en The International Seafood Trade. Woodhead Publishing Limited: CRC Press.

Bailey, C. M. (1988). The Social Consequences of Tropical Shrimp Mariculture Development. Ocean and Shoreline Management, 11, 31-44.

Barking, J. S. \& De Sombre, E. R. (2013). Saving Global Fisheries, Reducing Fishing Capacity to Promote Sustainability, The MIT Press.

Béné, C., Macfadyen, G. \& Allison, E. H. (2007). Increasing the Contribution of Small-Scale Fisheries to Poverty Alleviation and Food Security. Roma: FAO, Fisheries Technical Paper.

Béné, C., Lawton, R. \& Allison, E. (2010). Trade Matters in the Fight Against Poverty Narratives, Perceptions, and (Lack of) Evidence in the Case of Fish Trade in Africa. World Development, 38 (7), 933-954.

Bestor, T. C. (2004). Tsukiji, The Fish Market at the Center of the World. University of California Press.

Bostock, T., Greenhalgh, P. \& Kleih, U. (2004). Policy Research - Implications of Liberalization of Fish Trade for Developing Countries. Synthesis Report. Chatham, UK: Natural Resources Institute, University of Greenwich. Clarke, S. (2009). Understanding China's Fish Trade and Traceability Systems. A Traffic East Asia Report. Traffic East Asia.

Clover, C. (2006). The End of the Line. How Overfishing is Changing the World and What We Eat. Berkeley, Los Angeles: University of California Press.

Diaz, R. J. \& Rosenberg, R. (2008). Spreading Dead Zones and Consequences for Marine Ecosystems. Science, 321, 926-929.

Food and Agriculture Organization (FAO) (1984). Informe de la Conferencia Mundial de la FAO sobre Ordenación y Desarrollo Pesqueros, Roma.

Food and Agriculture Organization (FAO) (2003). Fisheries Management. The Ecosystem Approach to FIsheries, Roma: FAO, Technical Guidelines for Responsible Fisheries, No. 4, Supplement 2.

Food and Agriculture Organization (FAO) (2005). Fisheries and Aquaculture Topics. Small-Scale and Artisanal Fisheries. Topics Fact Sheets. Text by Jan Johnson, faO Fisheries and Aquaculture Department. Recuperado el 12 de agosto del 2014, de http://www.fao.org/fishery/topic/14753/en

Food and Agriculture Organization (FAO) (2007). International Sea Food Trade: Challenges and Opportunities. Roma: FAO United Nations.

Food and Agriculture Organization (FAO) (2008). El estado mundial de la pesca y la acuacultura a nivel mundial 2018, Roma: FAO.

Food and Agriculture Organization (FAO) (2012a). El estado mundial de la pesca y la acuacultura a nivel mundial 2012. Roma: FAO.

Food and Agriculture Organization (FAO) (2012b). Yearbook of Fishery Statistics. FAO Fisheries and Aquaculture Department. Recuperado el 12 de agosto del 2014, de ftp://ftp.fao.org/FI/STAT/summary/default.htm

Food and Agriculture Organization (FAO) (2014). El estado mundial de la pesca y la acuacultura a nivel mundial 2014. Roma: FAO.

Fernández Polanco, J. M. (2012). El mercado de acuacultura en el siglo XXI. Presente, pasado y tendencias de futuro. Madrid: Marcial Pons. 
García, S. M., Zerbi, A., Aliaume, C., Do-Chi, T. \& Lasserre, G. (2003). The Ecosystem Approach to Fisheries. Issues, Terminology, Principles, Institutional Foundations, Implementation and Outlook. Roma: FaO Fish Technical Paper, No. 443.

Hall, S. J., Dugan, P., Allison, E. H. \& Andrew, N. L. (2010). The End of the Line: Who is Most at Risk from the Crisis in Global Fisheries? Ambio, 39, 78-80.

Halpern, B. S., Walbridge, S., Selkoe, K. A., Kappel, C., Micheli, V., D’Agrosa, F., Bruno, C., Casey, J. F., Ebert, K. S., Fox, C., Fujita, H. E., Heinemann, R., Lenihan, D., Madin, H. S., Perry, E. M. P., Selig, M. T., Spalding, E. R., Steneck, M. \& Watson, R. R. (2008). A Global Map of human impact on Marine Ecosystems. Science, 319, 948-952.

Hilborn, R. (2012). Overfishing, What Everyone Needs to Know. Oxford University Press.

Kautsky, N., Berg, H., Folke, C., Larsson, J. \& Troell M. (1997). Ecological Footprint for Assessment of Resource Use and Development Limitations in Shrimp and Tilapia Aquaculture. Aquaculture Research, 28, 753-66.

Kris-Etherton, P. M., William, H. S. \& Appel, J. L. (2002). Fish Consumption, Fish Oil, Omega-3 Fatty Acids, and Cardiovascular Disease. AHA Scientific Statement, Circulation, 106, 2747-2757. doi: 10.1161/01. CIR.0000038493.65177.94

Kurien, J. (2005). Responsible Fish Trade and Food Security. Roma: FAO, Fisheries Tecnical Paper, 456.

Mialhe, F., Gunnel, Y. \& Mering, C. (2013). The Impacts of Shrimp Farming on Land Use, Employment and Migration in Tumbes, Northern Peru. Ocean and Coastal Management, 73, 1-12.

Mullon, C., Fréon, P. \& Cury, P. (2005). The Dynamics of Collapse in World Fisheries. Fish and Fisherie, 6,111-20.

Pauly, D., Alder, J., Bennett, E., Christensen, V., Tyedmers, P. \& Watson, R. (2003). The Future for Fisheries. Science, 302 (21), 1359-1361.

Primavera, J. H. (2006). Overcoming the Impacts of Aquaculture on the Coastal Zone. Ocean and Coastal Management, 49, 531-545.

Thorpe, A. (2004). Growth and Equity: Grounds for Inserting the Sector is PRSPS and NDPs. En Mainstreaming Fisheries into National Development and Poverty Reduction Strategies: Current Situation and Opportunities. Roma: FaO, Fisheries Circular, No. 997.

Wilson, J. R. \& Boncoeur, J. (2008). Microeconomic Efficiencies and Macroeconomic Inefficiencies: On Sustainable Fisheries Policies in Very Poor Countries. Oxford Development Studies, 36 (4), 339-460.

WorldFishing and Aquaculutre (2013). Japan Seeks to Boost Seafood Consumption. Recuperado el 9 de agosto del 2014 , de http://www.worldfishing.net/news101/regional-focus/japan-seeks-to-boost-seafood-consumption

WorldFishing Today (2009). Mitsubishi Hards Frozan Bluefin Tuna. At the Time of Crisis when the Fishing Stocks Decline Japanese firm Mitsubishi Hards Frozan Bluefin Tuna, 06-06-2009. Recuperado de http://www. worldfishingtoday.com/news/default.asp?nyId=3387.

Worm, B., Barbier, E. E., Beaumont, N. J., Duffy, E., Folke, C., Halpern, B. S., Jackson, J. B. C., Lotze, H. K., Micheli, F., Palumbi, S.R., Sala, E., Selkoe, K. A., Stachowicz, J. J. \& Watson, R. (2006). Impacts of Biodiversity Lost on Ocean Ecosystem Services. Science, 314, 787-790. 